\title{
Stress Whitening Morphology of Multiphase Polypropylene
}

\author{
Stanley P.Westphal
}

Westphal Beachside Consultants,Inc.

Satellite Beach, FL 32937

\begin{abstract}
Multiphase material is well known to appear white as if pigmented after a specimen has been subjected to a tensile stress. (1-2) After injection molding stress whitening has not been found to influence the final impact strength(3); it is an aesthetic problem. The impact from the knock-out pins frequently leaves a white mark after striking the part. However; it is a real problem when one material stress whitens at a lower impact load than another. This study was carried out to determine which factors controlled the minimum impact energy necessary to cause stress whitening.
\end{abstract}

In the test for stress whitening the injection molded ASTM Gardener Disc is subjected to a range of impact loading from 8.0 - 260 Joules. The first observed whitening is the Whitening Resistantance. Compression molding yields an entirely different behavior than the injection molded specimen. Compression molded parts with no oriented skin leave a hemisperical damage zone all the way to the exterior surface of maximum tensile stress. Injection molded specimens with a skin - core morphology leave a hemispherical damage zone in the spherulitic core with the maximum at the skin-core interface. Separate analyses have shown that the oriented skin is more crystalline with a higher modulus than the core. It also indicates that the infacial structure is greater inside the skin than the core.

Electron microscopy blocks were also analyzed. Microtomy was done at $-100 \mathrm{C}$. For general morphology studies the cryo-cut specimens were sonicated in n-heptane at 60 $\mathrm{C}$ for $20 \mathrm{~min}$. This procedure solubilizes the EPR particles and removes them from the matrix leaving a smooth but porous surface for analysis. For the special whitened samples there was no heptane sonication. Morphological analysis was done with an ISI-40 SEM at $15 \mathrm{KV}$. Image Analysis was done manually on a Zeiss Videoplan Image Analyzer. Image analysis is critical and yields both the concentration of the EPR phase and the size of the EPR particle. The average chord length was multiplied by 1.273 to get a better estimate of the actual average particle size(4) .

A normal impact copolymer after sonication in heptane is shown in Figure 1.A stress whitened sample viewed in the SEM clearly shows why the area appeared white. An EPR particle is clearly partially debonded from the polypropylene matrix and caused the light scattering. This is shown in Figure 2. Those microfibrils indicate the interface of the particle and matrix is a mixture of both polypropylene and ethylenepropylene molecules. Separate TEM studies on $\mathrm{RuO} 4$ stained samples indicate that the area around the particle is amorphous and there is some randomly distributed 
crystalline materials inside the particle. Structural factures which may be important include interfacial bond strength, EPR particle size and/or particle surface area, \# of particles/unit volume, oriented skin thickness and composition. Reactor conditions can be altered to vary the $\mathrm{C} 3 / \mathrm{C} 2$ gas mole ratio. Under these conditions there is a wide spectrum of ethylene-propylene products. The $\mathrm{C} 3 / \mathrm{C} 2$ ratio controls the composition of the EPR. The interfacial bond strength is much lower at high $\mathrm{C}_{3} / \mathrm{C}_{2}$ mole ratio's. The effect of the skin thickness occurs because the skin has a significantly higher modulus and crystallinity than the core.

\section{Figure 1- Normal NC-7 Etched Figure 2 -Stress Whitened Impact Copolymer \\ Impact Copolymer}
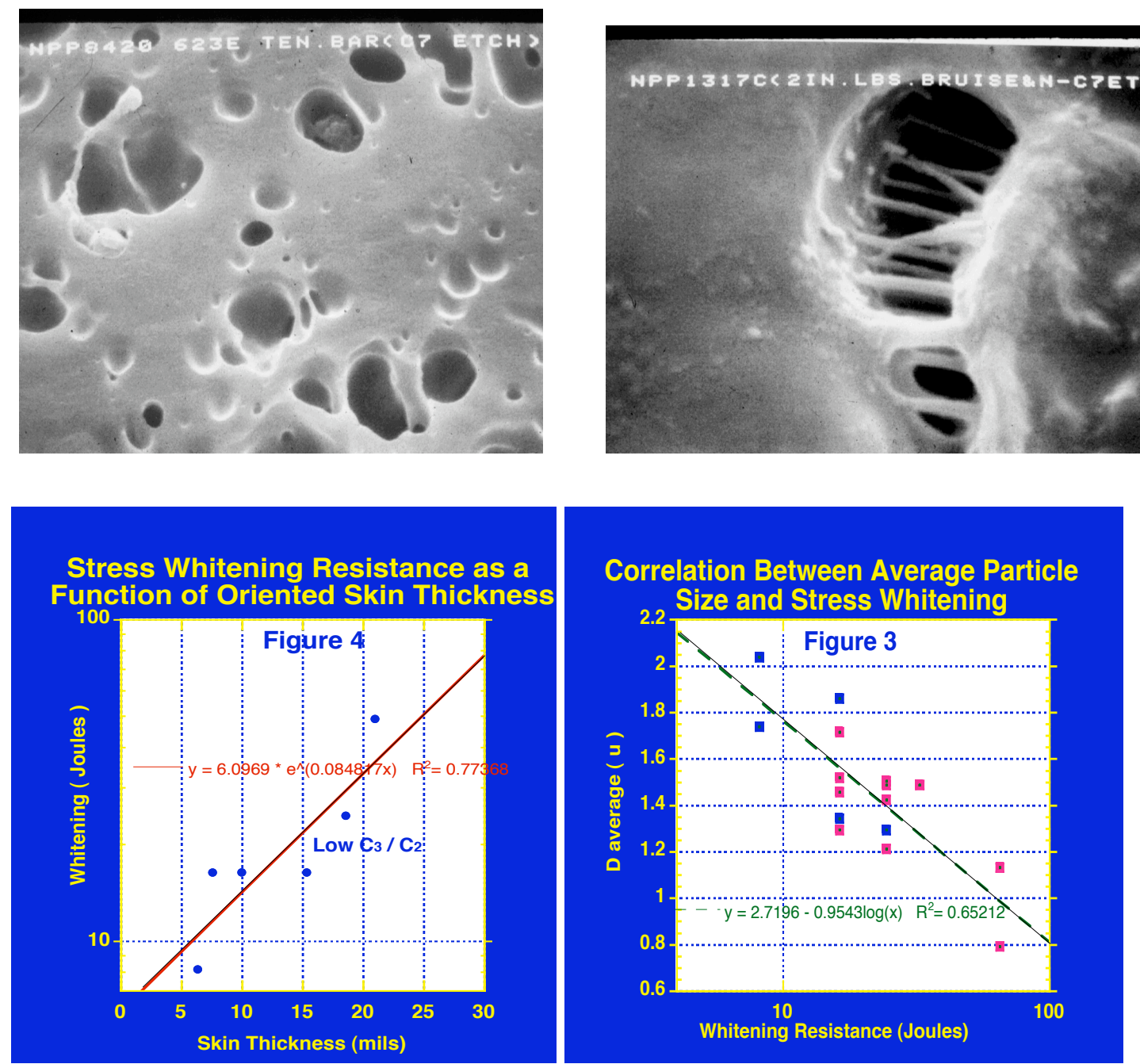

References

1.Kojima,Morio; Stress Whitening in Crystalline Propylene-Ethylene Block Copolymers, Sci.Phys.,B19(3).523-541 (1981).

2.Pressly,T., N.Jivraj, C.Bosnyak, G.Young, V.Tharp; Reduction of Stress Whitening in

Polypropylene Extrusion Blow Molded Bottles, Antec 2004.

3.Unpublished work by the author

4.Krumbein,W.C.; Journal.of Geology; Volume 43. 484(1935). 